\title{
STRUCTURAL AND KINEMATIC ANALYSIS OF EQUIPMENT TO CUT WEEDS BETWEEN PLANTS ON SAME ROW
}

\author{
Gabriel Gheorghe $^{1}$, Gheorghe Voicu ${ }^{2}$, Catalin Persu ${ }^{1}$ \\ ${ }^{1}$ National Institute of Research-Development for Machines and Installations Designed to Agriculture \\ and Food Industry, Romania; ${ }^{2}$ University Politehnica of Bucharest, Romania \\ gabrielvalentinghe@yahoo.com, ghvoicu_2005@yahoo.com, persucatalin@yahoo.com
}

\begin{abstract}
The analysis of the mechanisms studies the characteristics: structural, kinetic, kinetic and dynamic of some given mechanisms (the geometry of the analysed mechanism is known: the constant lengths and angles of the elements, the positions of the bases).Within the structural analysis, the number and type of kinematic elements and couplings, the number of the leading elements are determined and the structural and kinematic schemes of the mechanisms are divided into structural groups, respectively kinematic. The kinematic analysis studies the movement of the elements (positions, speeds, accelerations) without taking into account the forces acting on the elements. In the composition of a mechanism, kinetic elements and kinematic couplings are encountered. The structural analysis of a mechanism refers to: the number and shape of the elements, the order of their binding, the specification of the referenced element; number and type of kinematic couples (number of relative mobility and contact geometry); the degree of mobility of the mechanism; kinematic scheme, structural scheme, and graph attached to the mechanism; the decomposition of the mechanism into assurance groups. The paper presents the structural and kinematic analysis (positions, velocities, accelerations) of a mechanism aiming to drive the active bodies of equipment to cut weeds between plants on the same row that performs this work. The purpose of the structural analysis is to ensure the adequacy of the design from the point of view of safety and serviceability of the structure, to improve and validate the performance and reduce the need for costly prototypes or design changes later. The purpose of the analysis is to verify the design, from the point of view of the mechanism of operation of the system.
\end{abstract}

Keywords: structural analysis; kinematic analysis; cut weeds; soil.

\section{Introduction}

The analysis of the mechanisms studies the characteristics: structural, kinetic, kinetic and dynamic of some given mechanisms (the geometry of the analyzed mechanism is known: the constant lengths and angles of the elements, the positions of the bases).

Within the structural analysis, the number and type of kinematic elements and couplings and the number of the conducting elements are determined and the structural and kinematic schemes of the mechanisms are divided into structural groups, respectively kinematic. The kinematic analysis studies the movement of the elements (positions, speeds, accelerations) without taking into account the forces acting on the elements.

In the composition of a mechanism kinetic elements and kinematic couplings are encountered. The structural analysis of a mechanism refers to: the number and shape of the elements, the order of their binding, the specification of the reference element; number and type of kinematic couples (number of relative mobility and contact geometry); the degree of mobility of the mechanism; kinematic scheme, structural scheme and graph attached to the mechanism; the decomposition of the mechanism into structural groups $[1 ; 5]$.

Rigid elements (non-deformable, or considered non-deformable) consist of a single piece (machine member), or several machine members rigidly assembled together; examples of rigid elements: bars, cams, gears. Flexible (deformable) elements are used for remote transmission of movement and force; examples of flexible elements: cables, belts, chains. Simple kinematic elements have the $\operatorname{rank} j \leq 2$. The monetary elements have the $\operatorname{rank} j=1$, and the binary elements have the rank $j=2$. Complex kinematic elements have a rank greater than 2 . Ternary elements have a rank 3 , and those with a rank greater than 3 are called pollen elements.

The kinematic elements are represented graphically or numerically; the number 0 is generally assigned to the base, the other numbers being assigned to the movable elements. The graphic symbols for the main kinematic elements and couplings are set in standards (EN ISO 3952-1). The kinematic element, by which movement and force are introduced into the mechanism, is called the guiding element. The element, from which the desired forces and movements are obtained, is called the driven element. The element that has only rotating couplings is called a bar. The kinematic element that 
rotates completely around a fixed axis is called a crank, and the one that oscillates around a fixed axis within a rotation angle is called a rocker [2].

The kinematic element that is not directly connected to the base is called the connecting rod. The kinematic element that forms a translucent kinematic couple with a kinematic element and a rotating kinematic couple with another kinematic element is called a slide. The sliding stone or the skid represents the compact kinematic element that slides along a guide, and the guide represents the kinematic element that requires a translucent movement of the sliding stone.

The known methods for calculating the mobility of the mechanisms are grouped into two categories: the first is based on the system of kinematic constraint equations and the calculation of its rank, and the second is a fast method, which does not require writing these equations $[3 ; 8]$.

Within this work, the object of the experimental research was chosen: the weed extraction body of the intelligent pesticide equipment, equipment that was designed and manufactured within the INMA Bucharest.

The mechanism of actuation of the weed removal body between plants (Fig. 1) of the smart frying equipment consists of: 1 - central frame for gripping the mechanism, 2 - electric actuator for actuating the mechanism, 3 - three-point clamping system for transforming the movement from the forward direction of the actuator arm to the forward direction of the extirpation body between plants.

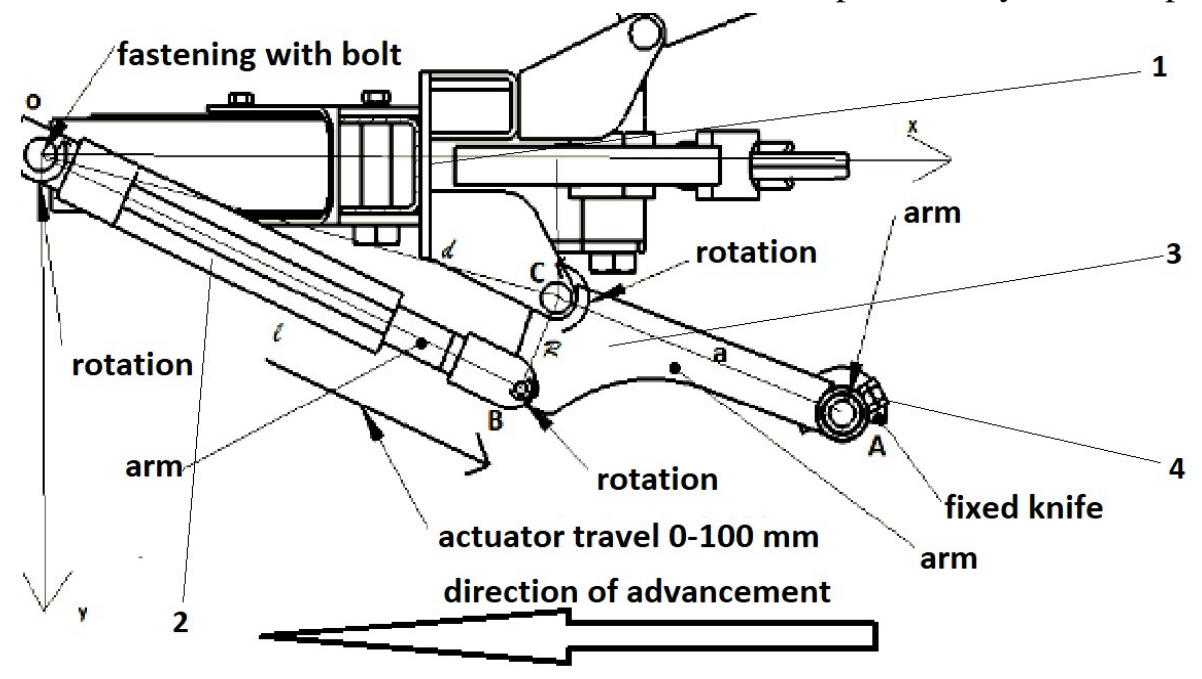

Fig. 1. Mechanism of action of the extraction body between plants

\section{Materials and methods}

\section{Freedom degree. Degree of mobility of the mechanism}

The degree of mobility $(M)$ of the mechanism or the degree of freedom $(L)$ of a kinematic chain represents the number of independent (coordinate) parameters needed to define the configuration of the mechanical system (the positions of all elements) at a given time, relative to a coordinate system attached to the base, respectively, to an element of the kinematic chain. The degree of mobility is used to verify the existence of a mechanism, because in a mechanism the condition must met: $M>0$ [4-6].

The most common formula used in the literature for rapid calculation of the overall degree of mobility of the mechanisms is the mobility criterion attributed to Cebishev Grubler-Kuţbah, which contains certain structural parameters of the mechanism [7;9].

$$
\begin{aligned}
& p=n+q \\
& q=p-n=p-(m-1)=p-m+1 \\
& M=6(m-1)-\sum_{i=1}^{p} c_{i} \\
& M=b(m-p-1)+\sum_{i=1}^{p} f_{i} \\
& M=\sum_{i=1}^{p} f_{i}-\sum_{j=1}^{q} b_{j}
\end{aligned}
$$


where $m$-number of elements of the mechanism, including the fixed element;

$p$ - number of kinematic couplings of the mechanism;

$f_{i}$ - connectivity of the kinematic coupling i (the number of degrees of freedom of the kinematic coupling), but it is the degree of constraint of the coupling $i$;

$q$ - number of independent contours; the number of independent contours of a mechanism, which has at least one element with the rank greater than 2 , which in the graph theory is called Euler's formula;

$b$ - number of degrees of freedom of the space in which the mechanism works (initially called mobility number);

$b_{j}$ - number of degrees of freedom of the space in which the closed chain $\mathrm{j}$ operates; the use of this initial definition of the mobility number, incorrectly formulated for the term $b_{j}$, results in incorrect results for the degree of mobility.

The theoretical study was applied using the constructive parameters of the equipment to cut weeds between plants on the same row, designed at INMA Bucharest (Fig. 2).

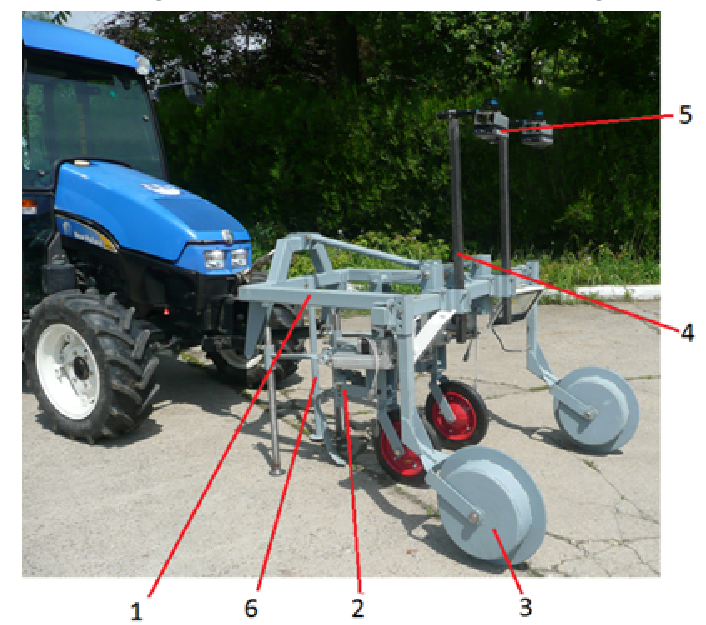

Fig. 2. Experimental model of the equipment to cut weeds between plants on the same row

The contradiction that arises in this case, and that we want to eliminate, is the following: improving the accuracy of calculating the degree of mobility for any mechanism, using the simplest possible relations (this means avoiding writing the kinematic constraint equations, which are complicated), with easy to set parameters. This contradiction will be eliminated by a strategy that uses the principles recommended by the methods of invention. The following principles were used for quick calculation of the mobility of the mechanisms: segmentation: the mobile elements of the mechanism, or the reference ones, with a rank greater than or equal to 2, are segmented so that only open kinematic chains exist. Prior to the kinematic analysis, the kinematic scheme of the mechanism was prepared (Fig. 3) [10].
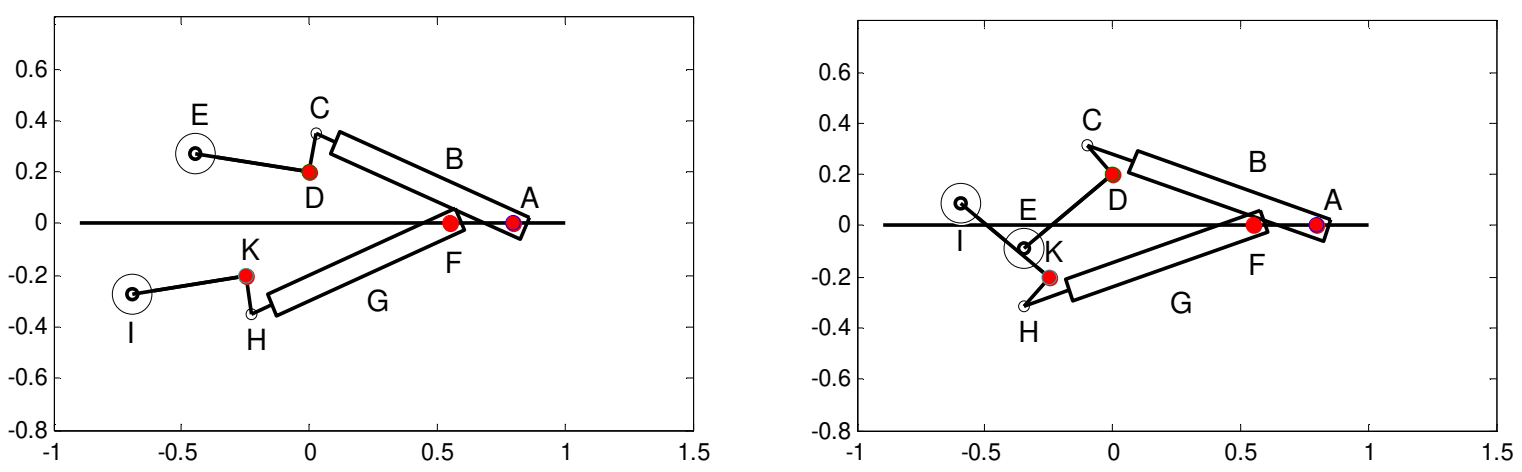

Fig. 3. Kinematic scheme of the mechanism 


\section{Program for kinematic analysis of the mechanism for cutting weeds between rows}

$\mathrm{cd}=0.15 ; \mathrm{de}=0.45 ; \mathrm{kl}=0.45 ; \mathrm{hk}=0.15$;

$\mathrm{a}=\left[\begin{array}{llllll}0.80 & 0 & 0 & 0 & 0 & 0\end{array}\right]$;

$\mathrm{d}=\left[\begin{array}{llllll}0 & 0.2 & 0 & 0 & 0 & 0\end{array}\right] ;$

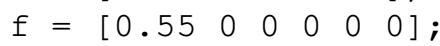

$\mathrm{k}=\left[\begin{array}{llllll}-0.25 & -0.2 & 0 & 0 & 0 & 0\end{array}\right] ;$

alfa $=\mathrm{pi} / 2$;

- functional input data:

$\varphi 1(1)=2.3 ; \varphi 3(1)=1.2 ; \varphi 4(1)=3.5 ; \varphi 6(1)=5$;

\% alte date

$\mathrm{n}=21$;

$\mathrm{n} 1=(\mathrm{n}-1) / 2$;

s0 $=0.85 ;$ cursa $=0.1$;

pas $=\operatorname{cursa} / \mathrm{n} 1$;

s1 $=\left[\begin{array}{lll}0 & 0.1 & 0\end{array}\right]$;

$\mathrm{s} 4=\left[\begin{array}{lll}0 & 0.1 & 0\end{array}\right]$;

for $i=1: n$

if $i<=n 1+1$

$\mathrm{s} 1(1)=\mathrm{s} 0+(i-1) *$ pas;

$\mathrm{s} 4(1)=\mathrm{s} 0+(i-1) *$ pas;

else

$\mathrm{s} 1(1)=\mathrm{s} 0+$ cursa $-(i-\mathrm{n} 1-1) *$ pas;

$\mathrm{s} 4(1)=\mathrm{s} 0+\operatorname{cursa}-(i-\mathrm{n} 1-1) *$ pas;

end

$[\varphi 3, \varphi 1, \mathrm{c}]=$ A2PVA_G $(\mathrm{d}, \mathrm{a}, \mathrm{s} 1, \mathrm{fi} 3, \mathrm{fi} 1, \mathrm{~cd}, \mathrm{~s} 0)$;

$[\mathrm{f} \varphi 6, \varphi 4, \mathrm{~h}]=\mathrm{A} 2 \mathrm{PVA} \mathrm{G}(\mathrm{k}, \mathrm{f}, \mathrm{s} 4, \mathrm{fi} 6, \mathrm{fi} 4, \mathrm{hk}, \mathrm{s} 0)$;

linel $(-0.9,1,0,0)$; hold;

e = A1RALFA_G(d, $\varphi$ 3, de, alfa); hold;

$1=$ AlRALFA_G $(\mathrm{k}, \varphi 6, \mathrm{kl},-\mathrm{alfa})$; hold;

$\mathrm{s}(\mathrm{i})=\mathrm{s} 1(1)$;

- Preparation of the painting for angles:

$\operatorname{vr} 1=[\varphi 1(1), \varphi 3(1), \varphi 4(1), \varphi 6(1)]$;

$\operatorname{vr2}=[\varphi 1(2), \varphi 3(2), \varphi 4(2), \varphi 6(2)]$;

$\operatorname{vr} 3=[\varphi 1(3), \varphi 3(3), \varphi 4(3), \varphi 6(3)]$;

$\operatorname{varungh}(i,:)=[i-1, \operatorname{vr} 1]$;

$\operatorname{varvit}(i,:)=[i-1, \operatorname{vr} 2]$;

$\operatorname{varacc}(i,:)=[i-1, \operatorname{vr} 3]$;

- Preparation of the figure for angles:

$\mathrm{flp}(\mathrm{i})=\varphi 1(1) ; \mathrm{f3p}(\mathrm{i})=\varphi 3(1) ; \mathrm{f} 4 \mathrm{p}(\mathrm{i})=\varphi 4(1) ; \mathrm{f} 6 \mathrm{p}(\mathrm{i})=\varphi 6(1) ;$

- Preparation of the figure for angular speeds:

$\mathrm{flv}(\mathrm{i})=\varphi 1(2) ; \mathrm{f3v}(\mathrm{i})=\varphi 3(2) ; \mathrm{f} 4 \mathrm{v}(\mathrm{i})=\varphi 4(2) ; \mathrm{f} 6 \mathrm{v}(\mathrm{i})=\varphi 6(2) ;$

- Preparation of the figure for angular accelerations:

$\mathrm{fla}(\mathrm{i})=\varphi 1(3) ; \mathrm{f3a}(\mathrm{i})=\varphi 3(3) ; \mathrm{f} 4 \mathrm{a}(\mathrm{i})=\varphi 4(3) ; \mathrm{f} 6 \mathrm{a}(\mathrm{i})=\varphi 6(3)$; plot (e (1), e (2),' o', l(1), l(2),' o', 'MarkerEdgeColor',' k', ...

'MarkerFaceColor',' 'w',' MarkerSize', 17);

$e=$ AlRALFA_G $(d, \varphi 3$, de, alfa $) ;$ hold;

$1=$ AlRALFA_G $(\mathrm{k}, \varphi 6, \mathrm{kl},-\mathrm{alfa})$; hold;

plot (a (1), a (2),' o' $, d(1), d(2), \prime^{\prime} o^{\prime}, f(1), f(2), o^{\prime}, k(1), k(2),{ }^{\prime} o^{\prime}, \ldots$

'MarkerfaceColor', ' r');

daspect $([1,1,1])$;

$\operatorname{axis}\left(\left[\begin{array}{lll}-1 & 1.5 & -1.1 .2]) \text {; }\end{array}\right.\right.$

pause $(0.1)$;

hold off;

end

- Variation diagrams of the angles according to s-figures (3):

plot $\left(s, f 1 p, s, f 1 p, k^{-\prime}, s, f 3 p, s, f 3 p, k^{\prime} x^{\prime}, \ldots\right.$ 


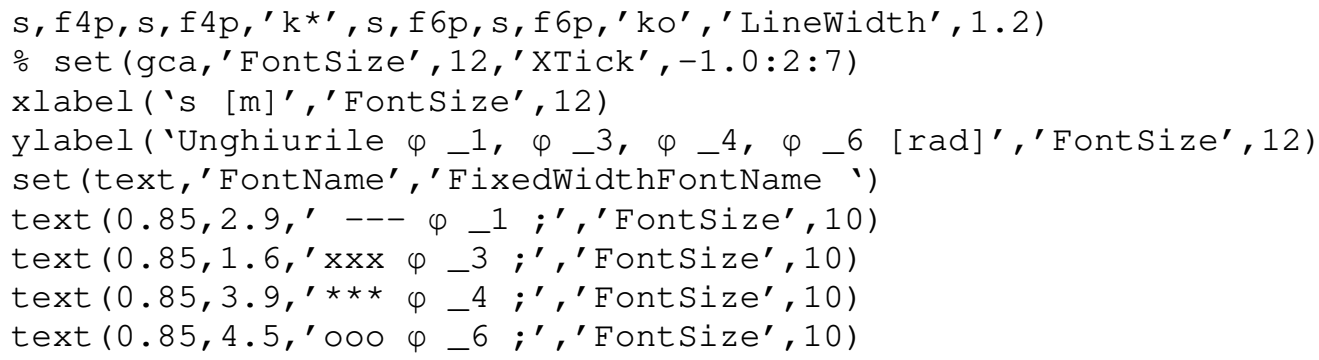

- Variation diagrams of the angular velocities according to s-figures(4):

plot $\left(s, f 1 v, s, f 1 v,{ }^{\prime} k-^{\prime}, s, f 3 v, s, f 3 v,{ }^{\prime} k x^{\prime}, \ldots\right.$

$\mathrm{s}, \mathrm{f} 4 \mathrm{v}, \mathrm{s}, \mathrm{f} 4 \mathrm{v}, \mathrm{\prime}^{*}{ }^{\prime}, \mathrm{s}, \mathrm{f} 6 \mathrm{v}, \mathrm{s}, \mathrm{f} 6 \mathrm{v},{ }^{\prime} \mathrm{ko} \mathrm{\prime}^{\prime},{ }^{\prime}$ LineWidth', 1.2$)$

xlabel ('s [m]','FontSize', 12)

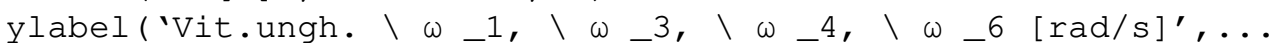

'FontSize', 12)

text $\left(0.86,0.2, \prime^{\prime}---\backslash \omega{ }^{\prime \prime},{ }^{\prime}\right.$ FontSize', 10$)$

text $\left(0.86,0.8,^{\prime} \mathrm{xxx} \backslash \omega{ }^{\prime}{ }^{\prime},{ }^{\prime}\right.$ FontSize', 10)

text $\left(0.86,-0.2,{ }^{\prime} * \star \star \star \omega 4^{\prime \prime},{ }^{\prime}\right.$ FontSize', 10$)$

text $\left(0.86,-0.9,^{\prime}\right.$ ooo $\backslash \omega{ }^{\prime} 6^{\prime},{ }^{\prime}$ FontSize', 10)

- Variation diagrams of the angular accelerations according to s-figures (5):

plot $\left(s, f 1 a, s, f 1 a,{ }^{\prime} k+{ }^{\prime}, s, f 3 a, s, f 3 a,{ }^{\prime} k{ }^{\prime}{ }^{\prime}, \ldots\right.$

$\mathrm{s}, \mathrm{f} 4 \mathrm{a}, \mathrm{s}, \mathrm{f} 4 \mathrm{a}, \mathrm{\prime}^{\prime} \mathrm{ko}$, s, ff6a, s, f6a, ' kx', ' LineWidth', 1.2)

xlabel ('s [m]','FontSize', 12)

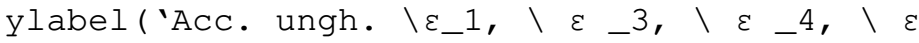

$6\left[\mathrm{rad} / \mathrm{s}^{\wedge} 2\right]^{\prime},{ }^{\prime}$ FontSize', 12)

text $\left(0.92,0.4, '+++\backslash \varepsilon{ }^{\prime} 1\right.$ ','FontSize', 10)

text $\left(0.92,1.1, '---\backslash \varepsilon_{-} 3\right.$ ','FontSize', 10)

text $\left(0.92,-0.45, '\right.$ oo \ $\backslash \varepsilon$ - $^{4}$ ','FontSize', 10)

text $\left(0.92,-1.1,{ }^{\prime} \operatorname{xxx} \backslash \varepsilon \_6\right.$ ','FontSize', 10)

- Numerical tables of the parameters of the mechanism elements:

xlswrite ('EX-unghiuri.xls', varungh);

xlswrite ('EX-vitezeungh.xls', varvit);

xlswrite('EX-accelerungh.xls', varvit);

\section{Results and discussion}

In analogy with the one-dimensional translation movement, for which we have used notions such as: linear coordinate $x$, linear velocity $\mathrm{v}$ and linear acceleration a, we describe the rotational motion of a body using: angular coordinate $\theta$ (Fig. 4), angular velocity $\omega$ (Fig. 5) and angular acceleration $\varepsilon$ (Fig. 6), defined in the same way as their correspondents from the translation movement.

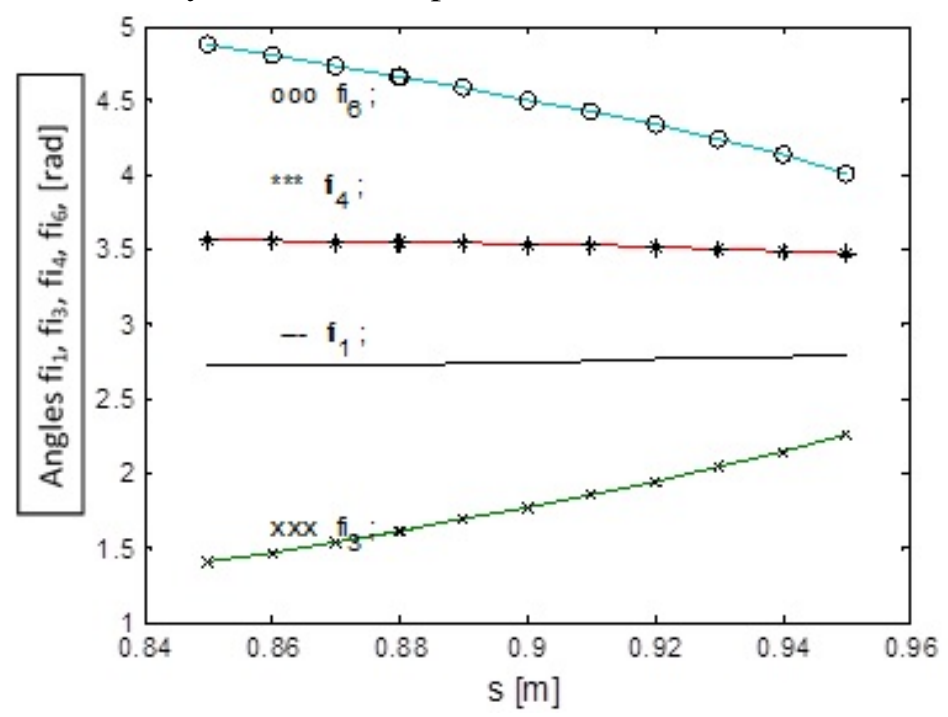

Fig. 4. Variation diagrams of angles 


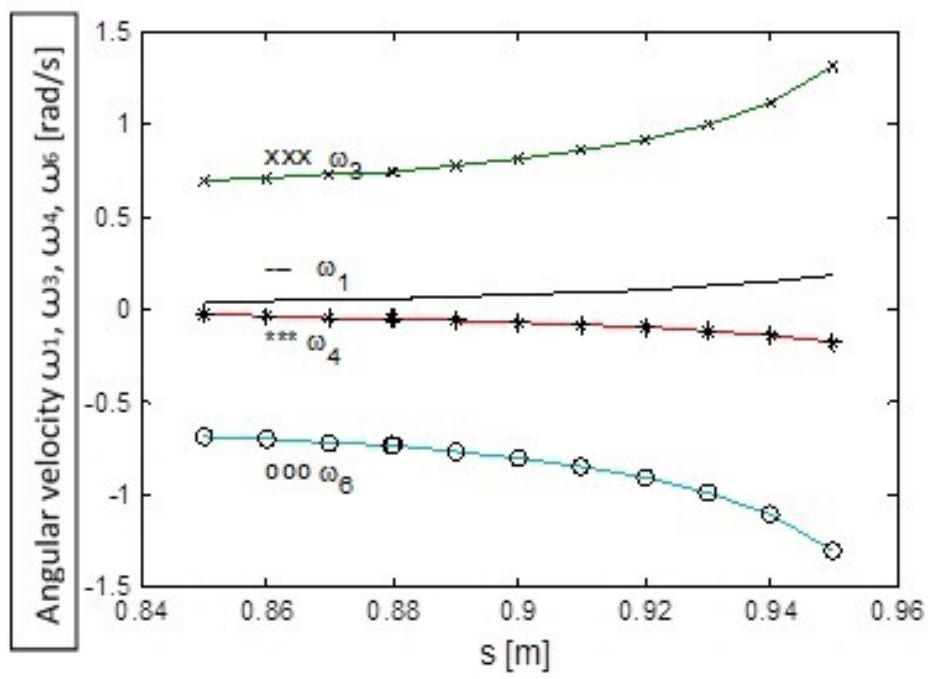

Fig. 5. Variation diagrams of angular velocities

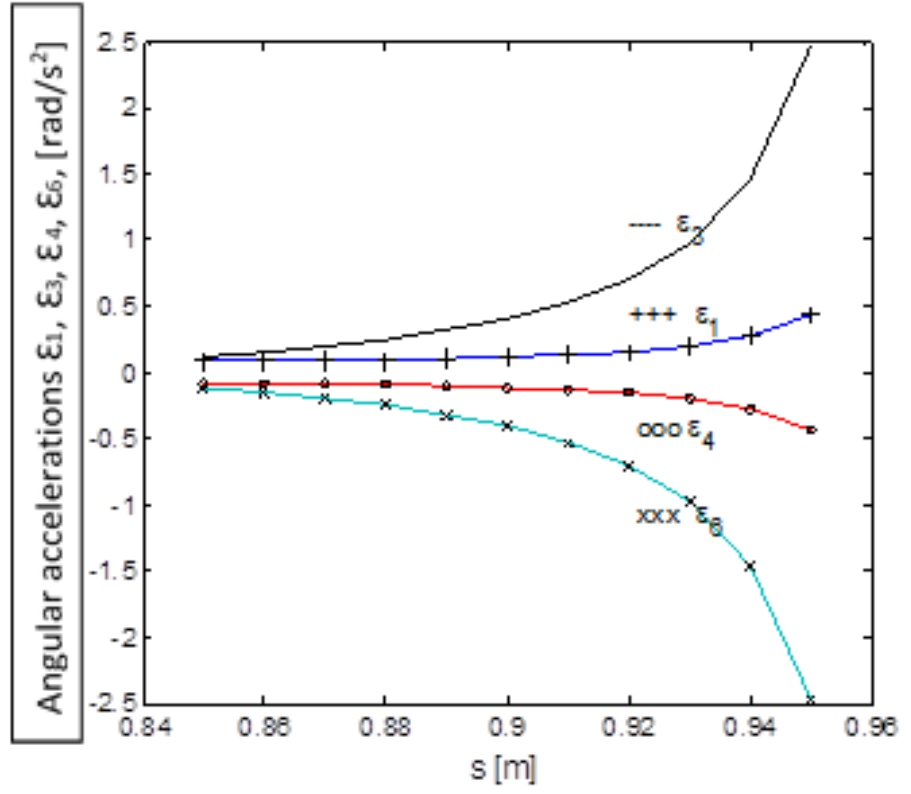

Fig. 6. Variation diagrams of angular accelerations

\section{Conclusions}

1. This analysis was performed to observe, if the system works as intended in the design and there are no blockages in the system.

2. Thanks to these analyses, we will know how each bar changes according to the others in real time and the next step is to find a mathematical formula to correlate the forward speed, the distance between the plants and the protection zone of the plant to make this mechanism work between plants in same row without any sensor.

3. Any moving mechanical system needs such analysis before it is executed to avoid certain execution problems, such as interference between elements.

\section{Acknowledgements}

This work was supported by a grant of the Romanian Research and Innovation Ministry, through Programme 1 - Development of the National Research-Development System, subprogramme 1.2 Institutional performance - Projects for financing excellence in RDI, contract no. 16PFE. 


\section{References}

[1] Moise V. Sinteza mecanismelor plane cu bare articulate aplicatii in MATLAB (Synthesis of plane mechanisms with articulated bars applications in MATLAB), Editura PRINTECH, 2018. (In Romanian)

[2] Artobolevski I.I. Théorie des Mécanismes et des Machines (Theory of Mechanisms and Machines). Ed. Mir, 1977. (In French).

[3] Duca C., Buium Fl. et al. Mechanisms, Gh. Asachi Publishing House, 2003.

[4] Moise V., Maican E., Moise Şt. I. Numerical methods in engineering, Bren Publishing House, 2003.

[5] Moise V., Simionescu I., Ene M. et al. Analysis of Applied Mechanisms, Printech Publishing House, 2008.

[6] Pelecudi Chr. The precision of the mechanisms, Publishing House of the Academy of RSR, 1975.

[7] Simionescu I., Moise V. Mechanisms, Technical Publishing House, 1999.

[8] Lucretia P., Moise S.I., Stefan V., Ungureanu L.M., Dudici L. "Structural and kinematic analysis of lifting and folding mechanism of mobile shutter", 18th International Scientific Conference "Engineering for Rural Development", Jelgava, Latvia, 2019. pp. 655-661.

[9] Pandrea N., Popa D., Stănescu N.D. Classical and Modern Approaches in the Theory of Mechanisms.

[10] Cretu S.M. "TRIZ method introduced to the calculation field", Procedia Engineering, 2011, volume 9, pp. 500-511.

[11]Popa P., Dudici L., Nedelcu A., Andreea-Catalina C., Daniel-Marian D. "Kinetostatic analysis of ratchet mechanism that acts on conveyor of manure spreading machine", 17th International Scientific Conference "Engineering for Rural Development”, Jelgava, Latvia, 2018. pp. 57-63. 